\title{
WEAK CONVERGENCE TO THE FIXED POINT OF AN ASYMPTOTICALLY NONEXPANSIVE MAP
}

\author{
S. C. BOSE
}

\begin{abstract}
It is proved that, in certain Banach spaces, any asymptotically nonexpansive and asymptotically regular map has the property that its iterates, applied to any point in the domain, give a sequence converging weakly to a fixed point.
\end{abstract}

Our object is to extend Opial's convergence theorem (Theorem 2 in [7]) to the case of asymptotically nonexpansive map. Suppose $K$ is a nonempty bounded closed convex subset of a Banach space $X$. A mapping $T: K \rightarrow K$ is called asymptotically nonexpansive [5] if for each $x, y \in K$

$$
\left\|T^{i} x-T^{i} y\right\| \leqslant k_{i}\|x-y\|, \quad i=1,2, \ldots,
$$

where $\left\{k_{i}\right\}$ is a fixed sequence of positive reals such that $k_{i} \rightarrow 1$ as $i \rightarrow \infty$. Existence of fixed points of such a mapping when $X$ is uniformly convex has been proved by Goebel and Kirk in [5].

In §1, we recall some basic definitions and known results. In §2, after Kirk we construct what we call the asymptotically central set of a sequence and observe some simple facts about it. Our main results are contained in $\$ 3$.

1. A mapping $T: K \rightarrow K$ is called nonexpansive if $\|T x-T y\| \leqslant\|x-y\|$ for any $x, y$ in $K$. It is called asymptotically nonexpansive if it satisfies (*) above. $T$ is asymptotically regular if for any $x \in K, T^{n} x-T^{n+1} x \rightarrow 0$, as $n \rightarrow \infty$. It is demiclosed if for any sequence $\left\{x_{n}\right\} \subset K, x_{n} \rightarrow x_{0}$ and $T x_{n} \rightarrow y_{0}$ $\Rightarrow T x_{0}=y_{0}$ where $\rightarrow$ denotes weak convergence. The modulus of convexity of $X$ is a function $\delta:[0,2] \rightarrow[0,1]$ defined by $\delta(\varepsilon)=\inf \left\{1-\frac{1}{2}\|x+y\|\right.$ : $\|x\| \leqslant 1,\|y\| \leqslant 1,\|x-y\| \geqslant \varepsilon\}$. It is known that $\delta$ is a nondecreasing function and is continuous on [0,2). It is also known [8], [9] that

$$
\begin{gathered}
\|x\| \leqslant d, \quad\|y\| \leqslant d, \\
\|x-y\| \geqslant \varepsilon \Rightarrow \frac{1}{2}\|x+y\| \leqslant\left(1-\delta\left(\frac{\varepsilon}{d}\right)\right) d .
\end{gathered}
$$

Opial [7] has shown that in a uniformly convex Banach space having weakly continuous duality mapping (or in a Hilbert space) if a sequence $\left\{x_{n}\right\}$ converges weakly to an $x_{0}$ then

$$
\lim _{n} \inf \left\|x_{n}-x_{0}\right\|<\lim _{n} \inf \left\|x_{n}-x\right\| \quad \forall x \neq x_{0} .
$$

Received by the editors April 14, 1977.

AMS (MOS) subject classifications (1970). Primary 47H10, 46B99; Secondary 54C05. 
It is easy to observe (see [2]) that the above inequality can be given an equivalent form in terms of lim sup:

$$
\lim _{n} \sup \left\|x_{n}-x_{0}\right\|<\lim _{n} \sup _{n}\left\|x_{n}-x\right\| \quad \forall x \neq x_{0} .
$$

2. Let $K$ be a nonempty bounded closed convex subset of a reflexive Banach space $X$ and let $\left\{x_{n}\right\}$ be any sequence in $K$. Following Kirk [6] and Edelstein [4] let us define the following:

$$
r(x)=\lim _{n} \sup _{n}\left\|x_{n}-x\right\|, \quad x \in X .
$$

This $r$ is a continuous function of $X$ into the reals (see Edelstein [3]).

$$
\begin{aligned}
\rho & =\rho_{K}\left(\left\{x_{n}\right\}\right)=\inf \{r(x): x \in K\}, \\
C_{0} & =\{x \in K: r(x)=\rho\} .
\end{aligned}
$$

$\rho$ is called the asymptotic radius of $\left\{x_{n}\right\}$ in $K$ and we prefer to call $C_{0}$ the asymptotically central set of $\left\{x_{n}\right\}$ in $K . C_{0}$ is a singleton if the space is uniformly convex (Proposition 2 below). In that case it is called asymptotic center.

Let $B_{n}(r)$ denote the closed ball of radius $r$ centered at $x_{n}$ and define

$$
C_{\varepsilon}=\bigcup_{i>1}\left(\bigcap_{n>i} B_{n}(\rho+\varepsilon)\right) \text {. }
$$

Proposition 1 [5]. $C_{0}=\bigcap_{\varepsilon>0}\left(K \cap \bar{C}_{\varepsilon}\right)$ and is a nonempty closed convex subset of $K$.

Proposition 2 [3]. If the space is uniformly convex then $C_{0}$ is a singleton.

The following lemma easily follows from Proposition 2 and the inequality $\left(0^{\prime}\right)$.

LEMMA 1. Let $K$ be a nonempty bounded closed convex subset of a uniformly convex Banach space having weakly continuous duality mapping. If a sequence $\left\{x_{n}\right\} \subset K$ converges weakly to a point $x_{0}$ then $x_{0}$ is the asymptotic center of $\left\{x_{n}\right\}$ in $K$.

In the proof of Theorem 2 in [1] we have observed that the space being uniformly convex if $T: K \rightarrow K$ is asymptotically nonexpansive, then the asymptotic center of $\left\{T^{n} x\right\}$ in $K$ for any $x \in K$ is a fixed point of $T$. We now prove:

3. LEMma 2. Let $K$ and $X$ be as in Lemma 1, T: $K \rightarrow K$ an asymptotically nonexpansive mapping. Suppose $x_{0}$ is the asymptotic center of the sequence $\left\{T^{n} x\right\}$ for some $x \in K$. If the weak limit $\xi_{0}$ of a subsequence $\left\{T^{n_{i}} x\right\}$ is a fixed point of $T$, then it must coincide with $x_{0}$ (which is a fixed point as remarked above).

Proof. Let $\rho$ and $\rho^{\prime}$ be the asymptotic radii respectively of $\left\{T^{n} x\right\}$ and $\left\{T^{n_{i}} x\right\}$. Clearly $\rho^{\prime} \leqslant \rho$. Since $\left\{T^{n_{i}} x\right\}$ converges weakly to $\xi_{0}$, by Lemma $1, \xi_{0}$ 
must be the asymptotic center of $\left\{T^{n_{i}} x\right\}$ in $K$, so that given any $\varepsilon>0$ we can choose an integer $i_{0}$ such that

$$
\left\|\xi_{0}-T^{n_{i 0}}\right\| \leqslant \rho^{\prime}+\varepsilon / 2 .
$$

Since $\xi_{0}$ is a fixed point of $T$ and $T$ is asymptotically nonexpansive we can choose an integer $J$ such that

$$
\left\|\xi_{0}-T^{n_{i_{0}}+j} x\right\| \leqslant k_{j}\left(\rho^{\prime}+\varepsilon / 2\right) \leqslant \rho^{\prime}+\varepsilon \leqslant \rho+\varepsilon \quad \text { for all } j \geqslant J .
$$

It follows therefore that $\lim \sup _{n}\left\|\xi_{0}-T^{n} x\right\|=\rho$ and $x_{0}$ being the unique point with this property we have $\xi_{0}=x_{0}$. The proof is complete.

Now we prove our convergence theorem.

THEOREM. Let $X$ be a uniformly convex Banach space having weakly continuous duality mapping and $K$ a nonempty bounded closed convex subset of $X$. Suppose $T: K \rightarrow K$ is asympotically nonexpansive and asymptotically regular. Then for any $x \in K$, the sequence $\left\{T^{n} x\right\}$ converges weakly to a fixed point of $T$.

Proof. We will show that the asymptotic regularity of $T$ makes every weak cluster point of $\left\{T^{n} x\right\}$ a fixed point of $T$. In view of the above lemma this would mean that all the weak cluster points of $\left\{T^{n} x\right\}$ coincide with the asymptotic center $x_{0}$ of $\left\{T^{n} x\right\}$ in $K$ (which is a fixed point) and would complete the proof.

Let us suppose that the subsequence $\left\{T^{n_{i}} x\right\}$ converges weakly to $\xi_{0}$. Then by Lemma $1, \xi_{0}$ will be the asymptotic center of $\left\{T^{n_{i}} x\right\}$ in $K$, let the asymptotic radius be $\rho$. By asymptotic regularity of $T,(I-T) T^{n} x \rightarrow 0$ as $i \rightarrow \infty$. Since $\left\{T^{n_{i}} x\right\}$ converges weakly to $\xi_{0}$, this implies $\left\{T^{n_{i}+1} x\right\}_{i=1}^{\infty}$ converges weakly to $\xi_{0}$. It follows in the same way that for any integer $r$ the sequence $\left\{T^{n_{i}+r} x\right\}_{i=1}^{\infty}$ converges weakly to $\xi_{0}$ and thus all these sequences have the same asymptotic center $\xi_{0}$ in $K$. We now claim that all these sequences have the same asymptotic radius $\rho$.

We have

$$
\begin{aligned}
\left\|\xi_{0}-T^{n_{i}+1} x\right\|-\left\|\xi_{0}-T^{n_{i}} x\right\| & \leqslant\left\|\left(\xi_{0}-T^{n_{i}+1} x\right)-\left(\xi_{0}-T^{n_{i}} x\right)\right\| \\
& =\left\|T^{n_{i}} x-T^{n_{i}+1} x\right\| \rightarrow 0 \text { as } i \rightarrow \infty
\end{aligned}
$$

by asymptotic regularity of $T$. Thus

$$
\lim \sup _{i}\left\|\xi_{0}-T^{n_{i}+1} x\right\|=\lim \sup _{i}\left\|\xi_{0}-T^{n_{i}} x\right\|=\rho
$$

and our claim follows.

We now prove that $\xi_{0}$ is a fixed point of $T$. If we can show that $T^{j} \xi_{0} \rightarrow \xi_{0}$ as $j \rightarrow \infty$, by continuity of $T$ this will mean $\xi_{0}$ is a fixed point of $T$, so let us suppose $T^{j} \xi_{0}$ does not converge to $\xi_{0}$. Then there is a $d>0$ and a sequence $\left\{j_{m}\right\}$ of integers such that

$$
\left\|\xi_{0}-T^{j_{m}} \xi_{0}\right\| \geqslant d \text { for all } m \text {. }
$$

By uniform convexity of the space, we may choose an $\varepsilon>0$ such that 
$(\rho+\varepsilon)[1-\delta(d /(\rho+\varepsilon))]<\rho$. Since all the sequences

$$
\left\{T^{n_{i}+r} x\right\}_{i=1}^{\infty}, \quad r=0,1,2, \ldots,
$$

have the same asymptotic center $\xi_{0}$ and the same asymptotic radius $\rho$, there exist integers $I=I(r)$ such that

$$
\left\|\xi_{0}-T^{n_{i}+r} x\right\| \leqslant \rho+\varepsilon / 2 \text { for all } i \geqslant I(r) .
$$

We have for any $m$,

$$
\left\|T^{j_{m}} \xi_{0}-T^{n_{i}+j_{m}} x\right\| \leqslant k_{j_{m}}\left\|\xi_{0}-T^{n_{i}} x\right\| \leqslant k_{j_{m}}(\rho+\varepsilon / 2) \text { for } i \geqslant I(0) .
$$

We choose an integer $M$ such that (as $k_{j} \rightarrow 1$ as $\left.j \rightarrow \infty\right) k_{j_{m}}(\rho+\varepsilon / 2) \leqslant \rho+\varepsilon$ for all $m \geqslant M$, so that we have

$$
\left\|T^{j_{m}} \xi_{0}-T^{n_{i}+j_{m}} x\right\| \leqslant \rho+\varepsilon \text { for all } i \geqslant I(0) \text { and all } m \geqslant M
$$

and from (1) we have

$$
\left\|\xi_{0}-T^{n_{i}+j_{m}} x\right\| \leqslant \rho+\varepsilon \quad \text { for } i \geqslant I\left(j_{m}\right) .
$$

Since $\left\|\xi_{0}-T^{j_{M}} \xi_{0}\right\| \geqslant d,(2)$ and (3) yield

$$
\left\|\frac{\xi_{0}+T^{j_{M}} \xi_{0}}{2}-T^{n_{i}+j_{M}}(x)\right\| \leqslant(\rho+\varepsilon)\left[1-\delta\left(\frac{d}{\rho+\varepsilon}\right)\right]<\rho
$$

for all $i \geqslant \max \left\{I(0), I\left(j_{M}\right)\right\}$. This is a contradiction in view of the fact that the sequence $\left\{T^{n_{i}+j_{M}} x\right\}_{i=1}^{\infty}$ has asymptotic radius $\rho$ in $K$. The proof of the theorem is therefore complete.

ACKNowledgement. I am indebted to my supervisor Dr. S. N. Patnaik for his going through the steps of this paper. I am also thankful to Mr. D. R. Joshi for his careful typing of the paper.

\section{REFERENCES}

1. S. C. Bose, On nonexpansive and asymptotically nonexpansive mappings (unpublished work).

2. E. Lami Dozo, Multivalued nonexpansive mappings and Opial's condition, Proc. Amer. Math. Soc. 38 (1973), 286-292.

3. M. Edelstein, Fixed point theorems in uniformly convex Banach spaces, Proc. Amer. Math. Soc. 44 (1974), 369-374.

4. Math. Soc. 78 (1972), 206-208.

5. K. Goebel and W. A. Kirk, A fixed point theorem for asymptotically nonexpansive mappings, Proc. Amer. Math. Soc. 35 (1972), 171-174.

6. W. A. Kirk, On nonlinear mappings of strongly semicontractive type, J. Math. Anal. Appl. 27 (1969), 409-412.

7. Z. Opial, Weak convergence of the sequence of successive approximations for nonexpansive mappings, Bull. Amer. Math. Soc. 73 (1967), 591-597.

8. L_L Lecture notes on nonexpansive and monotone mappings in Banach spaces, Center for Dynamical Systems, Brown University, Providence, R. I., 1967.

9. H. Schaefer, Uber die Methode sukzessiver Approximationen, Jber. Deutsch. Math.-Verein. 59 (1957), 131-140.

Department of Mathematics, Indian Institute of Technology, New Delhi 110029, India 